\title{
Insatisfacción corporal y calidad de vida: contribución a la ocurrencia de conductas alimentarias anómalas en administrativos bospitalarios
}

\author{
Luis Javier Bernardino-Coronado; Georgina Alvarez-Rayón; María Leticia Bautista-Díaz; Mayaro Ortega-Luyando; \\ Juan Manuel Mancilla-Díaz; Adriana Amaya-Hernández
}

How to cite this article:

Bernardino-Coronado, L.J., Alvarez-Rayón, G., Bautista-Díaz, M.L., Ortega-Luyando, M., Mancilla-Díaz, J.M. \& Amaya-Hernández, A. (2020). Body dissatisfaction and quality of life: contribution to the occurrence of anomalous eating behaviors in administrative personnel in hospitals. Acta Colombiana de Psicología, 23(1), 28-40. doi: http://www. doi.org/10.14718/ACP.2020.23.1.3

Recibido, marzo 20/2019; Concepto de evaluación, mayo 4/2019; Aceptado, junio 11/2019

Luis Javier Bernardino-Coronado

Universidad Nacional Autónoma de México, Ciudad de México, México

ORCID: https://orcid.org/0000-0002-6224-5199

Georgina Alvarez-Rayón

Universidad Nacional Autónoma de México, Ciudad de México, México

ORCID: https://orcid.org/0000-0001-7106-7032

María Leticia Bautista-Díaz

Universidad Autónoma del Estado de Hidalgo, Hidalgo, México

ORCID: https://orcid.org/0000-0003-1154-1737

Mayaro Ortega-Luyando

Universidad Nacional Autónoma de México, Ciudad de México, México ORCID: https://orcid.org/0000-0003-4665-3894

Juan Manuel Mancilla-Díaz

Universidad Nacional Autónoma de México, Ciudad de México, México ORCID: https://orcid.org/0000-0001-7259-3667

Adriana Amaya-Hernández

Universidad Nacional Autónoma de México, Ciudad de México, México ORCID: https://orcid.org/0000-0003-0011-4959

\section{Resumen}

\begin{abstract}
El objetivo del presente estudio consistió en evaluar la capacidad predictiva de variables como el índice de masa corporal (IMC), la imagen corporal, el afecto positivo y negativo, las actitudes hacia la gente obesa y la calidad de vida (CV) sobre las conductas alimentarias anómalas del personal administrativo del sector salud en la Ciudad de México, además de conocer las diferencias entre los sexos y del IMC en las variables de estudio. En total, participaron 181 trabajadores administrativos, divididos según su IMC (normopeso, sobrepeso, obesidad), que completaron una hoja de datos generales y cuestionarios de autorreporte. En general, se encontró que la insatisfacción de la imagen corporal, aunada a la percepción de vitalidad y bienestar físico, podría explicar en parte la presencia del comportamiento alimentario anómalo, el cual favorece a la ganancia excesiva de peso corporal. Adicionalmente, se observaron diferencias significativas entre los sexos en el afecto positivo, además de diferencias entre el grupo con obesidad y el grupo normopeso en insatisfacción corporal, afecto negativo, funcionamiento físico, rol físico y funcionamiento social.

Palabras clave: obesidad, servicios de salud, conducta alimentaria anómala, imagen corporal, calidad de vida.
\end{abstract}

\footnotetext{
* Grupo de Investigación en Nutrición, Universidad Nacional Autónoma de México, FES-Iztacala, División de Investigación y Posgrado. Av. de los Barrios \#1, Los Reyes Iztacala, Tlalnepantla, C. P.: 54090, México. Tel.: +52 (55) 56231333 ext.39737. aamayah@hotmail.com Agradecemos al PAPIIT (UNAM) por el financiamiento con registro IA302618 otorgado a la Dra. Adriana Amaya-Hernández.
} 


\title{
Body dissatisfaction and quality of life: contribution to the occurrence of anomalous eating behaviors in administrative personnel in hospitals
}

\begin{abstract}
The purpose of this study involved assessing the predictive capacity of the body mass index (BMI), body image, positive and negative affect, attitudes toward obese individuals and quality of life (QL) related to the anomalous eating behaviors of administrative personnel of Mexico City's health sector; aside from knowing gender differences and BMI in study variables. A total of 181 administrative employees took part in the study, divided according to their BMI (normal weight, overweight, obese) who filled out a personal data sheet and self-report questionnaires. Findings of this study indicate that body dissatisfaction, along with the perception of vitality and physical well-being may partially explain the presence of the anomalous eating behaviors that promotes excessive weight gain. Additionally, significant differences in positive affect were observed between sexes, aside from differences between the obese and normal weight groups regarding body dissatisfaction, negative affect, physical performance, physical role and social performance.

Key words: obesity, health care services, anomalous eating behaviors, body image, quality of life.
\end{abstract}

\section{Introducción}

Estudios realizados en México han evaluado diferentes indicadores de salud en profesionales que prestan sus servicios en el sector salud —como médicos, enfermeras, laboratoristas, entre otros - $y$ han encontrado que entre el 38 y el $75 \%$ de los participantes presentaba problemas de sobrepeso u obesidad (Nanguce, Ceballo-León \& ÁlvarezReyes, 2015; Navarro et al., 2005; Nieves-Ruíz, HernándezDurán \& Aguilar-Martínez, 2011; Palacios-Rodríguez, Munguía-Miranda \& Avila-Leyva, 2006). Este hallazgo es llamativo porque, debido a que México se encuentra entre los primeros lugares en la prevalencia de obesidad adulta $\mathrm{e}$ infantil (Organización para la Cooperación y el Desarrollo Económicos [OCDE], 2018), uno de los objetivos del sector salud es dar soporte a campañas de concientización sobre los problemas de salud derivados del exceso de peso.

A nivel mundial, se estima la existencia de aproximadamente 1400 millones de adultos con sobrepeso y 500 millones de adultos con obesidad (Organización Mundial de la Salud [OMS], 2014); y, respecto a México, la Encuesta Nacional de Salud y Nutrición (ENSANUT) de Medio Camino (Shamah, Cuevas, Rivera \& Hernández, 2016) indicó que la prevalencia combinada de sobrepeso y obesidad en adultos mayores de 20 años era de $72.5 \%$ para el año 2016, lo que representó un incremento de 1.3 puntos porcentuales con respecto a la ENSANUT de 2012 (71.2\%) (Gutiérrez et al., 2012).

Ahora bien, entre los múltiples factores de riesgo asociados al sobrepeso y la obesidad, Guzmán, del Castillo y García (2010) destacaron dos factores ambientales por su fuerte vínculo con estas condiciones de salud: la falta de actividad física y los hábitos nocivos del estilo de vida.

Con respecto al primer factor, según datos del módulo de práctica deportiva y ejercicio físico del Instituto Nacional de Estadística y Geografía (INEGI, 2016), el $58 \%$ de la población general en México es sedentaria o inactiva, y, al considerar el género, hay un mayor porcentaje de estos problemas en las mujeres (63\%). Asimismo, en un informe más reciente (INEGI, 2017a) se encontró que, de la población mexicana laboralmente activa, un importante porcentaje realiza sus actividades a nivel terciario, lo cual quiere decir que dichas personas, debido a su sedentarismo laboral, solo gastan entre 1.4 y $1.8 \mathrm{kcal}$ por minuto; esto en contraste con trabajadores de sectores relacionadas con la agricultura, la minería o la construcción — considerados de nivel primario y secundario- que llegan a demandar hasta $10 \mathrm{kcal}$ por minuto de trabajo (Wanjeck, 2005).

Es con este panorama que se hace relevante el segundo factor, los hábitos nocivos del estilo de vida (Guzmán et al., 2010), ya que, con tales prácticas laborales, en las grandes ciudades la población general se ve en la necesidad de alimentarse fuera del hogar, lo cual repercute generalmente en el consumo de alimentos poco saludables, altos en grasa y con bajo valor nutrimental (García, 2012). Como consecuencia, este hábito termina propiciando una constante ganancia de peso y, eventualmente, que las personas con sobrepeso u obesidad practiquen métodos de pérdida de peso poco efectivos, como lo son las dietas restrictivas o los ayunos prolongados, los cuales dan pie a patrones de 
alimentación anómalos (Reséndiz, Hernández, Sierra \& Torres, 2015) que propician aún más la ganancia de peso.

Una vez que estas personas llegan al sobrepeso o la obesidad, algunas de las consecuencias negativas que más repercusión en la salud tienen en ellas son:

1. Las enfermedades no transmisibles (ENT) relacionadas con la obesidad, como lo son la hipertensión arterial, las dislipidemias, la diabetes mellitus y el síndrome de apnea/ hipopnea obstructiva del sueño (Campos-Nonato, Barquera \& Aguilar, 2012). De estas, es importante considerar que la diabetes es una de las principales causas de muerte en México (INEGI, 2017b), y que, por tanto, esta representa un costo elevado para el sector salud.

2. La modificación corporal dada por la ganancia de peso, ya que con esta se promueve la insatisfacción corporal derivada del desagrado o inconformidad con la imagen corporal —en la que se incluyen componentes perceptuales, cognitivos, afectivos y conductuales-, y que se asocia a la adopción de conductas nocivas que atentan contra la salud (Thompson, Heinberg, Altabe \& Tantleff-Dunn, 1999; Thompson \& Smolak, 2001). Con respecto a esto, en diversos estudios se ha encontrado que un mayor IMC se asocia a una mayor insatisfacción corporal en adultos (Casillas-Estrella, Montaño-Castrejón, Reyes-Velázquez, Bacardi-Gascón \& Jiménez-Cruz, 2006); que al comparar mujeres con obesidad con mujeres con normopeso se observa que las primeras reportan mayores niveles de insatisfacción corporal (Gilbert-Diamond, Baylin, Mora-Plazas \& Villamor, 2009); y que la condición de obesidad pone a las personas en un mayor riesgo de sufrir discriminación, lo cual contribuye tanto a acentuar la imagen corporal negativa como a la disminución de la autoestima, a la estigmatización y, en definitiva, a la afectación de su bienestar psicológico (Bell \& Morgan, 2000).

3. Y la disminución de la calidad de vida, definida como la percepción que un individuo tiene en cuanto a su posición en la vida tanto en el contexto de su cultura y del sistema de valores en los que vive como en relación con sus objetivos, expectativas, estándares e intereses (OMS, 2017). En este aspecto, algunos estudios han argumentado que el sobrepeso y la obesidad predicen una percepción menor de la calidad de vida, y que esta, además, parece disminuir conforme incrementa el peso corporal (de Zwaan et al., 2009; Sarwer, Lavery \& Spitzer, 2012).

Finalmente, el sobrepeso y la obesidad representan una de las principales problemáticas de salud a nivel mundial, ya que sus repercusiones se ven reflejadas en el desempeño cotidiano de la persona que la padece. Cabe mencionar que algunos estudios han demostrado que este padecimiento se ve incluso reflejado en el personal de instituciones de salud (Nanguce et al., 2015; Navarro et al., 2005; NievesRuíz et al., 2011; Palacios-Rodríguez et al., 2006), ya que estas personas tienen que cumplir con horarios de trabajo excesivos que limitan el tiempo para seguir una adecuada alimentación, y esto a su vez puede estar mermando la calidad del servicio prestado a los pacientes.

Teniendo lo anterior en cuenta, conocer qué favorece las conductas alimentarias anómalas del personal de instituciones de salud ayudará a crear programas preventivos para disminuir la incidencia del sobrepeso y la obesidad y aumentar las conductas saludables. Por ello, el objetivo del presente estudio fue evaluar la capacidad predictiva de variables como el IMC, la imagen corporal, el afecto positivo y negativo, las actitudes hacia la gente obesa y la calidad de vida en las conductas alimentarias anómalas, además de conocer las diferencias entre los sexos y del IMC en las variables de estudio.

\section{Método}

\section{Participantes}

La muestra fue no probabilística, y de tipo intencional, integrada por 181 trabajadores de la Secretaría de Salud -105 mujeres de 17 a 66 años $(M=39.27, D E=11.31)$ y 76 hombres de 21 a 72 años $(M=43.43, D E=12.72)$-, todos residentes de la zona metropolitana de la Ciudad de México. Más de la mitad de la muestra reportó educación a nivel superior, donde el $48.07 \%$ dijo ser de nivel licenciatura, y el $18.78 \%$, de posgrado (véase Tabla 1 ).

\section{Instrumentos}

Hoja de datos generales. Se realizó una hoja de registro con la finalidad de obtener los datos sociodemográficos $\longrightarrow$ p. ej., edad, sexo, estado civil—y de las condiciones generales de salud $\longrightarrow$ p. ej., presencia de enfermedades, uso de sustancias nocivas para la salud, actividad física, entre otras - de los participantes.

Test de Actitudes Alimentarias (EAT-26, por sus siglas en inglés; Garner, Olmsted, Bohr \& Garfinkel, 1982). Este instrumento evalúa actitudes y comportamientos característicos de los trastornos alimentarios mediante 26 reactivos con seis opciones de respuesta tipo Likert. En México, Franco, Solorzano, Díaz e Hidalgo-Rassmussen (2016) y Vázquez et al. (2017) evaluaron su consistencia interna, que resultó ser buena $(\alpha=.91)$, mientras que en la muestra del presente estudio se obtuvo un coeficiente alfa de .83 . 
Cuestionario de Imagen Corporal (BSQ, por sus siglas en inglés; Cooper, Taylor, Cooper \& Fairburn, 1987). Este cuestionario evalúa la imagen corporal mediante 34 reactivos con seis opciones de respuesta tipo Likert, y fue adaptado por Vázquez et al. (2011) para la población mexicana, en donde se reportó una excelente consistencia interna $(\alpha=.98)$. En la muestra del presente estudio se obtuvo un alfa de .96 .

Escala de Afecto Positivo y Negativo (PANAS, por sus siglas en inglés; Watson, Clark \& Tellegen, 1988). Esta escala evalúa el estado de ánimo mediante un listado de 20 adjetivos ( 10 de afecto positivo y 10 de afecto negativo) con cinco opciones de respuesta tipo Likert, y está compuesta por dos modalidades, una dirigida a evaluar el estado —última semana y hoy - y otra, el rasgo — usualmente- Robles y Páez (2003) evaluaron sus propiedades psicométricas en población mexicana, donde se confirmó su estructura bifactorial con coeficientes de consistencia interna de .85 (estado) y .90 (rasgo) para afecto positivo, y de .81 (estado) y .85 (rasgo) para afecto negativo. En la muestra del presente estudio se obtuvo de alfa de .91 para estado y rasgo en afectividad positiva, y de .87 (estado) y .88 (rasgo) para afectividad negativa.

Escala de Actitudes Hacia la Gente Obesa (ATOP, por sus siglas en inglés; Allison, Basile \& Yuker, 1991).

Este instrumento evalúa las percepciones y actitudes hacia las personas con obesidad por medio de 20 reactivos con seis opciones de respuesta tipo Likert. Allison et al. (1991), reportaron un alfa de .80 en el estudio original, mientras que en la muestra del presente estudio se obtuvo un alfa de .74 .

Encuesta de Salud (SF-36, por sus siglas en inglés; Stewart, Hays \& Ware, 1988). El SF-36 evalúa la calidad de vida relacionada con la salud, y consiste una encuesta compuesta por 36 reactivos divididos en ocho subescalas funcionamiento físico, rol físico, dolor corporal, percepciones generales de salud, vitalidad, funcionamiento social, rol emocional y salud mental - y dos indicadores globales — calidad física y calidad mental—. Se realizó una adaptación de la encuesta a población mexicana (Zúniga, Carrillo-Jiménez, Fos, Gandek \& Medina-Moreno, 1999), en donde se obtuvo coeficientes de consistencia interna adecuados, entre .70 y .84 . En la muestra del presente estudio se hallaron coeficientes alfa entre .66 y .89 .

Índice de Masa Corporal (IMC). Esteíndice se calcula por medio del autorreporte de peso y estatura de los participantes. En este estudio se consideraron los criterios propuestos por el Instituto Mexicano del Seguro Social (IMSS, 2017) para categorizar el IMC, es decir, normopeso (18.5-24.9), sobrepeso (25-29.9), obesidad nivel I (30-34.9), obesidad nivel II (35-39.9) y obesidad nivel III ( $\geq 40)$.

\section{Procedimiento}

Dado que se trata de una investigación transversal de tipo no experimental, se presentó el protocolo de investigación -que cuenta con la aprobación de la comisión de ética de la Facultad de Estudios Superiores Iztacala-UNAM (CE/ FESI/062018/1256) — a las autoridades de ocho Unidades Administrativas de la Secretaría de Salud. Una vez aprobada la aplicación, se contactó a los participantes para explicarles el propósito del estudio, momento en que se solicitó la firma del consentimiento informado y se dieron las instrucciones para contestar la hoja de datos generales y los cinco cuestionarios. Durante la aplicación, un investigador estuvo atento para aclarar dudas respecto a los cuestionarios y se formaron grupos de cinco a diez personas, dependiendo del espacio de cada Unidad y de la disponibilidad de los trabajadores. A los participantes que obtuvieron puntuaciones altas en uno o más cuestionarios, o bien su IMC fue indicador de sobrepeso $\mathrm{u}$ obesidad, se les dio una breve plática informativa sobre las consecuencias de su estado de salud y se les canalizó con un psicólogo o nutriólogo.

\section{Análisis de datos}

Para los análisis estadísticos se empleó el paquete estadístico SPSS (versión 20). Inicialmente, se realizó un análisis descriptivo de la muestra — datos que se presentan en términos de porcentajes - ; después, se realizó la prueba de Shapiro-Wilk — que demostró que las variables de estudio no tenían normalidad, excepto la de conductas alimentarias anómalas, por lo cual se optó por emplear pruebas no paramétricas - ; posteriormente, para las diferencias entre sexos se utilizó la U de Mann Whitney, mientras que para identificar diferencias en las variables evaluadas según el IMC se empleó la prueba de Kruskal Wallis; adicional a esto, para obtener la asociación entre variables se hizo uso de la r de Spearman; y, por último, para conocer las variables que explican las conductas alimentarias anómalas se realizó un análisis de regresión lineal múltiple - la significancia estadística se determinó con un nivel de $p \leq .05-$.

\section{Resultados}

\section{Análisis descriptivo}

Con respecto al IMC, dos terceras partes de la muestra $(67.95 \%)$ presentaban sobrepeso u obesidad, de las cuales casi la mitad (49.72\%) presentó sobrepeso y $18.23 \%$ 
obesidad (nivel I o II), siendo los hombres quienes registraron porcentajes más altos que las mujeres - no obstante, estas diferencias no fueron estadísticamente significativas $\left(X^{2}=1.79, p>.05\right)$ - (véase Tabla 1$)$.

En cuanto al uso de sustancias nocivas para la salud, el $23.20 \%$ mencionó consumir tabaco, mientras que el $41.98 \%$ dijo consumir alcohol. Del total de la muestra, el $41.98 \%$ consideró tener problemas de alimentación y el $51.93 \%$ de sobrepeso u obesidad; en ambos las mujeres reportaron porcentajes más altos que los hombres. Por otra parte, el $33.70 \%$ refirió haber padecido sobrepeso u obesidad en alguna etapa de su vida, mientras que el $39.22 \%$ mencionó tener uno o más familiares que padecen sobrepeso u obesidad; para ambos casos los hombres reportaron porcentajes más altos que las mujeres. Finalmente, el $31 \%$ de los participantes refirió practicar algún deporte, mayormente los hombres; en tanto que el $42 \%$ dijo haberse involucrado en programas de reducción de peso, en su mayoría mujeres (véase Tabla 2).

Por último, 40 participantes ( $22 \%$ ) mencionaron padecer una enfermedad crónica; la más reportada fue la hipertensión (6.63\%), seguida por la diabetes $(3.15 \%) \mathrm{y}$ la gastritis $(1.66 \%)$.
Comparación según el sexo y las categorías del IMC

Por una parte, se encontraron diferencias significativas entre sexos solo en la variable de afecto positivo (estado), siendo los hombres quienes obtuvieron puntuaciones más altas $(U=3132.50 ; p<.05)$; y, por otra, diferencias entre los grupos del IMC en las variables de insatisfacción corporal, afecto negativo (estado y rasgo), funcionamiento físico, rol físico, funcionamiento social y salud mental. De acuerdo con la prueba de Dunn (post-hoc), en la mayoría de las variables las diferencias se ubicaron entre el grupo con obesidad y el grupo con normopeso (véase Tabla 3).

\section{Asociación entre variables}

Por medio del análisis de asociación entre variables se obtuvieron coeficientes significativos - de bajos a altosen los tres grupos del IMC. En los grupos con sobrepeso y con obesidad hubo mayor número de coeficientes significativos, principalmente entre las subescalas del SF-36 y las del PANAS, el ATOP y la insatisfacción corporal.

Las correlaciones altas y significativas en los tres grupos fueron entre las subescalas de los instrumentos; no obstante, se puede destacar la correlación entre la insatisfacción

Tabla 1.

Características sociodemográficas de la muestra

\begin{tabular}{lcccccc}
\hline & \multicolumn{2}{c}{ Mujeres $(n=105)$} & \multicolumn{2}{c}{ Hombres $(n=76)$} & \multicolumn{2}{c}{ Total $(N=181)$} \\
\hline Nivel de estudios & Frecuencia & $\%$ & Frecuencia & $\%$ & Frecuencia & $\%$ \\
$\quad$ Secundaria & & & & & & \\
Preparatoria & 11 & 10.48 & 0 & 0 & 11 & 6.08 \\
Licenciatura & 23 & 21.90 & 12 & 15.79 & 35 & 19.34 \\
Posgrado & 43 & 40.95 & 44 & 57.89 & 87 & 48.07 \\
Sin respuesta & 20 & 19.05 & 14 & 18.43 & 34 & 18.78 \\
Indice de masa corporal & 8 & 7.62 & 6 & 7.89 & 14 & 7.73 \\
Normopeso & & & & & & \\
Sobrepeso & 39 & 37.14 & 19 & 25 & 58 & 32.05 \\
Obesidad nivel I & 50 & 47.62 & 40 & 52.63 & 90 & 49.72 \\
Obesidad nivel II & 13 & 12.38 & 12 & 15.79 & 25 & 13.81 \\
Ingreso familiar mensual & 3 & 2.86 & 5 & 6.58 & 8 & 4.42 \\
Nivel alto & & & & & & \\
Nivel medio alto & 7 & 6.67 & 10 & 13.16 & 17 & 9.39 \\
Nivel medio típico & 3 & 2.86 & 9 & 11.84 & 12 & 6.64 \\
Nivel medio emergente & 22 & 20.95 & 20 & 26.32 & 42 & 23.20 \\
Nivel bajo emergente & 18 & 17.14 & 16 & 21.05 & 34 & 18.78 \\
Nivel bajo extremo & 28 & 26.67 & 7 & 9.21 & 35 & 19.34 \\
Sin respuesta & 18 & 17.14 & 10 & 13.16 & 28 & 15.47 \\
\hline & 9 & 8.57 & 4 & 5.26 & 13 & 7.18 \\
\hline
\end{tabular}


Tabla 2.

Características con relación a hábitos alimentarios y de salud en la muestra

\begin{tabular}{|c|c|c|c|c|c|c|}
\hline & \multicolumn{2}{|c|}{ Mujeres $(n=105)$} & \multicolumn{2}{|c|}{ Hombres $(n=76)$} & \multicolumn{2}{|c|}{ Total $(N=181)$} \\
\hline & Frecuencia & $\%$ & Frecuencia & $\%$ & Frecuencia & $\%$ \\
\hline \multicolumn{7}{|l|}{ Uso de sustancias } \\
\hline Tabaco & 22 & 20.95 & 20 & 26.31 & 42 & 23.20 \\
\hline Alcohol & 41 & 39.04 & 35 & 46.05 & 76 & 41.98 \\
\hline Drogas & 0 & 0 & 0 & 0 & 0 & 0 \\
\hline \multicolumn{7}{|l|}{ Problemas } \\
\hline Alimentarios & 50 & 47.61 & 26 & 34.21 & 76 & 41.98 \\
\hline Sobrepeso/obesidad & 59 & 56.19 & 35 & 46.05 & 94 & 51.93 \\
\hline \multicolumn{7}{|l|}{ Antecedentes sobrepeso/obesidad } \\
\hline Vida & 35 & 33.33 & 26 & 34.21 & 61 & 33.70 \\
\hline Familia & 40 & 38.09 & 31 & 40.78 & 71 & 39.22 \\
\hline Práctica de deporte & 37 & 35.23 & 40 & 52.63 & 77 & 42.54 \\
\hline Programas de reducción de peso & 41 & 39.04 & 15 & 19.73 & 56 & 30.93 \\
\hline
\end{tabular}

corporal y las conductas alimentarias anómalas para los grupos de normopeso $(r=.71)$, sobrepeso $(r=.59)$ y obesidad $(r=.67)$ (véase Tabla 4).

\section{Predicción de conductas alimentarias anómalas}

Dado que la prueba de Kruskal Wallis demostró la inexistencia de diferencias significativas entre los grupos del IMC en la variable de conductas alimentarias anómalas, se decidió realizar el análisis estadístico de predicción considerando la muestra completa. Cabe señalar que los datos cumplieron con los supuestos que esta prueba requiere, es decir, independencia de errores —estimada con el estadístico de Durbin-Watson (1.973) - , homocedasticidad, normalidad, linealidad y no colinealidad —de 1.000 a 1.182, estimada con el factor de inflación de la varianza (FIV)-. De acuerdo con el diagnóstico de caso, se eliminaron seis casos que excedían las tres desviaciones típicas.

El modelo de regresión lineal múltiple explicó el $49.6 \%$ de la varianza de las conductas alimentarias anómalas a partir de la insatisfacción corporal y la calidad de vida, específicamente a partir del funcionamiento físico, el rol físico y la vitalidad (véase Tabla 5).

\section{Discusión}

El objetivo del presente estudio fue evaluar la capacidad predictiva de variables como el IMC, la imagen corporal, el afecto positivo y negativo, las actitudes hacia la gente obesa y la calidad de vida en las conductas alimentarias anómalas, además de conocer las diferencias entre los sexos y del IMC en las variables de estudio.

Entre las diversas variables que pueden estar asociadas a la génesis o mantenimiento de la obesidad se encuentra la presencia de conductas alimentarias anómalas, y esto es consistente con los resultados de la presente investigación, ya que en ellos se observa que la insatisfacción corporal, aunada a la percepción de vitalidad y bienestar físico, podría explicar en parte la presencia del comportamiento alimentario anómalo, lo cual podría convertirse en un ciclo vicioso del mantenimiento de la condición de exceso de peso. Algunas de las posibles explicaciones a este hallazgo son, por una parte, el ideal de belleza que ha sido establecido por la sociedad, en el que se dicta que una persona es bella si es delgada (Guzmán et al., 2010); y, por otra, los problemas que una persona enfrenta al realizar sus actividades cotidianas debido a su cuerpo. Estas dos condiciones pueden tener como consecuencia la presencia de insatisfacción corporal y la necesidad de remediar dicha situación, de lo cual puede surgir una aparente salida fácil que resulte en la realización de conductas alimentarias anómalas, como la dieta restrictiva y el subsecuente aumento de la ingestión de alimento.

Con respecto al sexo, en el presente estudio no se observaron diferencias en la insatisfacción corporal, lo cual contrasta con estudios previos (Bully \& Elosua, 2011; Mäkinen, Puukko-Viertomies, Lindberg, Siimes \& Aaalberg, 2012; Mellor, Fuller-Tyszkiewicz, McCabe \& Ricciardelli, 
Tabla 3.

Comparación entre sexo y entre grupos del IMC en las variables de estudio

\begin{tabular}{|c|c|c|c|c|c|c|c|c|c|}
\hline & $\begin{array}{c}\text { Mujeres } \\
(n=105) \\
\text { Rango } \\
\text { promedio }\end{array}$ & $\begin{array}{c}\text { Hombres } \\
(n=76) \\
\text { Rango } \\
\text { promedio }\end{array}$ & $p$ & $d$ & $\begin{array}{c}\text { Normopeso } \\
(n=62) \\
\text { Rango } \\
\text { promedio }\end{array}$ & $\begin{array}{l}\text { Sobrepeso } \\
(n=88) \\
\text { Rango } \\
\text { promedio }\end{array}$ & $\begin{array}{c}\text { Obesidad } \\
(n=31) \\
\text { Rango } \\
\text { promedio }\end{array}$ & $p$ & $d$ \\
\hline Conductas alimentarias anómalas & 91.26 & 90.64 & .937 & 0.01 & 90.31 & 84.73 & 110.18 & .066 & 0.28 \\
\hline $\begin{array}{l}\text { Insatisfacción corporal } \\
\text { Afección positiva }\end{array}$ & 96.24 & 83.76 & .114 & 0.37 & $74.98^{\mathrm{a}}$ & $93.16^{\mathrm{a}, \mathrm{b}}$ & $116.90^{\mathrm{b}}$ & .001 & 0.53 \\
\hline Rasgo & 85.52 & 98.57 & .098 & 0.25 & 97.03 & 86.47 & 74.98 & .172 & 0.19 \\
\hline Estado & 82.83 & 102.28 & .014 & 0.37 & 99.77 & 88.67 & 80.08 & .196 & 0.17 \\
\hline Afección negativa & & & & & & & & & \\
\hline Rasgo & 87.97 & 95.19 & .359 & 0.14 & $82.22^{\mathrm{a}}$ & $89.65^{\mathrm{a}, \mathrm{b}}$ & $112.39^{b}$ & .030 & 0.34 \\
\hline Estado & 86.50 & 97.21 & .174 & 0.20 & $85.16^{\mathrm{a}}$ & $87.32^{\mathrm{a}, \mathrm{b}}$ & $113.11^{\mathrm{b}}$ & .034 & 0.33 \\
\hline $\begin{array}{l}\text { Actitud hacia gente obesa } \\
\text { SF-36 }\end{array}$ & 86.17 & 97.68 & .144 & 0.22 & 82.69 & 93.94 & 99.27 & .271 & 0.12 \\
\hline $\mathrm{FF}$ & 87.58 & 95.72 & .281 & 0.15 & $104.25^{\mathrm{a}}$ & $90.48^{\mathrm{a}, \mathrm{b}}$ & $65.98^{b}$ & .002 & 0.49 \\
\hline $\mathrm{RF}$ & 90.63 & 91.51 & .876 & 0.02 & $99.77^{\mathrm{a}}$ & $88.95^{\mathrm{a}, \mathrm{b}}$ & $79.27^{\mathrm{b}}$ & .036 & 0.33 \\
\hline $\mathrm{DC}$ & 91.89 & 89.78 & .746 & 0.04 & 92.66 & 91.68 & 85.76 & .753 & 0.18 \\
\hline PGS & 90.65 & 91.48 & .916 & 0.01 & 96.40 & 92.38 & 76.29 & .202 & 0.17 \\
\hline $\mathrm{V}$ & 86.48 & 97.24 & .170 & 0.20 & 94.35 & 94.98 & 73.00 & .107 & 0.24 \\
\hline FS & 92.47 & 88.97 & .641 & 0.07 & $104.82^{\mathrm{a}}$ & $89.39^{\mathrm{a}, \mathrm{b}}$ & $67.92^{\mathrm{b}}$ & .003 & 0.47 \\
\hline $\mathrm{RE}$ & 88.57 & 94.36 & .292 & 0.11 & 98.64 & 84.39 & 94.48 & .053 & 0.30 \\
\hline SM & 85.31 & 98.86 & .085 & 0.26 & $92.44^{\mathrm{a}, \mathrm{b}}$ & $98.35^{\mathrm{a}}$ & $67.27^{\mathrm{b}}$ & .017 & 0.38 \\
\hline
\end{tabular}

Nota. Encuesta de salud (SF-36); funcionamiento físico (FF); rol físico (RF); dolor corporal (DC); percepción general de salud (PGS); vitalidad (V); funcionamiento social (FS); rol emocional (RE); salud mental (SM). $g l=2$.

Tabla 4.

Coeficientes de correlación entre las variables de estudio por grupos del IMC

\begin{tabular}{|c|c|c|c|c|c|c|c|c|c|c|c|c|c|c|c|c|}
\hline & 1 & 2 & 3 & 4 & 5 & 6 & 7 & 8 & 9 & 10 & 11 & 12 & 13 & 14 & 15 & 16 \\
\hline \multicolumn{17}{|c|}{ Normopeso $(n=62)$} \\
\hline 1. CAA & - & & & & & & & & & & & & & & & \\
\hline 2. IC & $.71 * *$ & - & & & & & & & & & & & & & & \\
\hline 3. APR & -.12 & -.23 & - & & & & & & & & & & & & & \\
\hline 4. APE & -.15 & $-.34 * *$ & $.84 * *$ & - & & & & & & & & & & & & \\
\hline 5. ANR & $.39 * *$ & $.45^{* *}$ & -.08 & -.12 & - & & & & & & & & & & & \\
\hline 6. ANE & $.26^{*}$ & $.43 * *$ & -.02 & -.12 & $.79 * *$ & - & & & & & & & & & & \\
\hline 7. ATOP & -.07 & .05 & .09 & -.05 & .03 & .04 & - & & & & & & & & & \\
\hline 8. FF & .01 & -.06 & $.42 * *$ & $.44 * *$ & .01 & .01 & -.21 & - & & & & & & & & \\
\hline 9. $\mathrm{RF}$ & -.04 & -.08 & -.11 & -.02 & -.12 & -.13 & -.09 & .12 & - & & & & & & & \\
\hline 10. DC & .02 & .15 & .01 & -.01 & -.03 & .05 & $-.28 *$ & .14 & -.01 & - & & & & & & \\
\hline 11. PGS & .03 & .13 & .23 & $.27^{*}$ & -.17 & -.01 & -.02 & $.32 *$ & .01 & $.29 *$ & - & & & & & \\
\hline 12. V & .03 & -.15 & $.40 * *$ & $.38 * *$ & -.09 & -.13 & $.28^{*}$ & $.34 * *$ & .01 & -.17 & $.27 *$ & - & & & & \\
\hline 13. FS & -.09 & -.04 & .17 & .16 & -.17 & -.07 & -.01 & $.36^{* *}$ & .06 & $.36^{* *}$ & $.59 * *$ & .25 & - & & & \\
\hline 14. RE & -.03 & .02 & -.02 & -.07 & -.20 & -.17 & -.13 & -.12 & .22 & .06 & .20 & -.11 & -.07 & - & & \\
\hline 15. SM & -.04 & -.17 & $.35^{* *}$ & $.40 * *$ & $-.26^{*}$ & $-.28 *$ & .13 & $.32 *$ & .12 & .11 & $.33^{*}$ & $.55^{* *}$ & $.53^{* *}$ & .01 & - & \\
\hline \multicolumn{17}{|c|}{ Sobrepeso $(n=88)$} \\
\hline 1. CAA & - & & & & & & & & & & & & & & & \\
\hline 2. IC & $.59 * *$ & - & & & & & & & & & & & & & & \\
\hline
\end{tabular}


Continuación

\begin{tabular}{|c|c|c|c|c|c|c|c|c|c|c|c|c|c|c|c|}
\hline 3. APR & -.08 & $-.34 * *$ & - & & & & & & & & & & & & \\
\hline 4. APE & -.11 & $-.35 * *$ & $.83 * *$ & - & & & & & & & & & & & \\
\hline 5. ANR & .16 & $.42 * *$ & $-.23 *$ & $-.22 *$ & - & & & & & & & & & & \\
\hline 6. ANE & $.22 *$ & $.46^{* *}$ & -.17 & -.24 & $.82 * *$ & - & & & & & & & & & \\
\hline 7. ATOP & $.27^{*}$ & .26 & -.03 & -.04 & .14 & $.23^{*}$ & - & & & & & & & & \\
\hline 8. FF & .10 & -.16 & .21 & $.23 *$ & -.09 & -.14 & -.08 & - & & & & & & & \\
\hline 9. $\mathrm{RF}$ & .04 & .03 & .10 & .08 & .01 & .05 & -.03 & $.50 * *$ & - & & & & & & \\
\hline 10. DC & -.04 & -.21 & .07 & -.03 & -.15 & $-.26^{*}$ & -.14 & .07 & -.19 & - & & & & & \\
\hline 11. PGS & .06 & $-.37 *$ & $.28 * *$ & $.26^{*}$ & $-.39 * *$ & $-.40^{*}$ & -.10 & $.41 * *$ & $.25^{*}$ & .12 & - & & & & \\
\hline 12. V & -.08 & $-.45 * *$ & $.30 * *$ & $.29 * *$ & $-.48 * *$ & $-.45 * *$ & -.11 & $.28 * *$ & .09 & $.22 *$ & $.36^{* *}$ & - & & & \\
\hline 13. FS & -.10 & $-.27 *$ & .26 & $.29 * *$ & $-.47 * *$ & $-.48 * *$ & -.15 & $.41^{* *}$ & $.30 * *$ & .15 & $.51 * *$ & $.47 * *$ & - & & \\
\hline 14. RE & -.08 & -.17 & .16 & $.24 *$ & $-.36 * *$ & $-.49 * *$ & -.13 & $.31 * *$ & $.31 * *$ & .18 & $.32 * *$ & $.43 * *$ & $.55^{* *}$ & - & \\
\hline 15. SM & -.13 & $-.48 * *$ & $.43^{* *}$ & $.46^{* *}$ & $-.60 * *$ & $-.60 * *$ & -.20 & $.28^{* *}$ & .14 & .13 & $.44^{* *}$ & $.75^{* *}$ & $.55^{* *}$ & $.46^{* *}$ & - \\
\hline \multicolumn{16}{|c|}{ Obesidad $(n=31)$} \\
\hline 1. CAA & - & & & & & & & & & & & & & & \\
\hline 2. IC & $.67 * *$ & - & & & & & & & & & & & & & \\
\hline 3. APR & -.03 & -.22 & - & & & & & & & & & & & & \\
\hline 4. APE & -.10 & -.31 & $.88 * *$ & - & & & & & & & & & & & \\
\hline 5. ANR & .16 & $.39 *$ & -.21 & -.25 & - & & & & & & & & & & \\
\hline 6. ANE & .28 & $.49 * *$ & -.15 & -.29 & $.88^{* *}$ & - & & & & & & & & & \\
\hline 7. ATOP & $.40^{*}$ & $.40 *$ & -.20 & -.29 & .30 & $.44^{*}$ & - & & & & & & & & \\
\hline 8. FF & $-.48 * *$ & $-.55 * *$ & $.50 * *$ & $.56^{* *}$ & -.33 & $-.43^{*}$ & $-.52 * *$ & - & & & & & & & \\
\hline 9. $\mathrm{RF}$ & $-.47 * *$ & $-.41 *$ & $.46^{*}$ & $.37 *$ & $-.47 * *$ & $-.41^{*}$ & -.22 & $.64 * *$ & - & & & & & & \\
\hline 10. DC & -.07 & .07 & .04 & -.03 & -.04 & .01 & -.12 & -.03 & -.20 & - & & & & & \\
\hline 11. PGS & -.34 & -.30 & $.54 * *$ & $.54 * *$ & -.22 & -.26 & -.16 & $.70 * *$ & $.59 * *$ & .01 & - & & & & \\
\hline 12. V & -.27 & -.36 & $.54 * *$ & $.53 * *$ & $-.54 * *$ & $-.56^{* *}$ & -.19 & .35 & $.63^{* *}$ & .11 & $.52 * *$ & - & & & \\
\hline 13. FS & $-.60 * *$ & $-.53 * *$ & .21 & .28 & -.30 & $-.45^{*}$ & $-.65 * *$ & $.81 * *$ & $.45^{*}$ & .14 & $.45^{*}$ & .26 & - & & \\
\hline 14. RE & -.13 & -.24 & .26 & $.42 *$ & $-.37 *$ & -.30 & -.22 & .18 & $.42 *$ & -.10 & .01 & .33 & .06 & - & \\
\hline 15. SM & $-.39 *$ & $-.45^{*}$ & .23 & .26 & $-.68 * *$ & $-.64 * *$ & -.31 & $.49 * *$ & $.67 * *$ & .06 & $.38^{*}$ & $.60 * *$ & $.46^{*}$ & $.49 * *$ & - \\
\hline
\end{tabular}

Nota. Conductas alimentarias anómalas (CAA); insatisfacción corporal (IC); afección positiva rasgo (APR); afección positiva estado (APE); afección negativa rasgo (ANR); afección negativa estado (ANE); actitud hacia gente obesa (ATOP); funcionamiento físico (FF); rol físico (RF); dolor corporal (DC); percepción general de salud (PGS); vitalidad (V); funcionamiento social (FS); rol emocional (RE); salud mental (SM); índice de masa corporal (IMC). ${ }^{*} p<.05,{ }^{* *} p<.01$.

Tabla 5.

Predictores de conductas alimentarias anómalas en empleados administrativos del sector salud $(N=175)$

\begin{tabular}{lccccccc}
\hline & $\mathrm{R}^{2}$ & $\Delta \mathrm{R}^{2}$ & $\mathrm{R}^{2}$ ajustada & $\mathrm{B}$ & $\beta$ & $t$ & $p$ \\
\hline Paso 1 & .447 & .447 & .444 & & & & \\
Insatisfacción corporal & & & & 0.30 & 0.67 & 11.82 & .000 \\
Paso 2 & .508 & .061 & .496 & & & & \\
Insatisfacción corporal & & & & 0.34 & 0.76 & 13.05 & .000 \\
Funcionamiento físico & & & 0.54 & 0.16 & 2.34 & .021 \\
Rol físico & & & -1.52 & -0.14 & -2.18 & .030 \\
Vitalidad & & & 0.83 & 0.21 & 3.53 & .001 \\
\hline
\end{tabular}


2010) en donde se ha reportado que las mujeres presentan mayor insatisfacción corporal y se involucran en métodos y conductas asociadas a la pérdida de peso o modificación corporal (Brechan \& Kvalem, 2015; McGuinness \& Taylor, 2016). Una posible explicación a este hallazgo es que, como lo han sustentado investigaciones previas, los mensajes encaminados a la estética corporal impactan a ambos sexos, mientras que la diferencia entre ellos radica en el deseo de un cuerpo esbelto en las mujeres y el de un cuerpo fitness en los hombres (Shaheen, Kumar, Dev, Parkash \& Rai, 2016).

En este estudio, los hombres presentaron un porcentaje mayor de sobrepeso y obesidad, sin embargo, las diferencias no fueron estadísticamente significativas. Este dato que no concuerda con lo reportado por la ENSANUT Medio Camino (Shamah et al., 2016), ya que en ella se menciona que las mujeres presentan tasas más altas de estos padecimientos.

Otro hallazgo contradictorio en esta investigación es que un mayor porcentaje de hombres realiza actividad física, pero a la vez son ellos quienes presentan mayor sobrepeso y obesidad. Con esto se refutaría la idea de que la práctica de cualquier actividad física, por mínima que sea, permite mantenerse en forma y con un nivel de salud óptimo (Bollado, 2014). Teniendo esto en cuenta, se sugiere retomar lo reportado por la OMS (2010), en donde se menciona que la actividad física beneficia la salud de quien la práctica solo si se realiza de forma regular, es decir, de 30 a 60 minutos en al menos cinco días a la semana. Este dato resalta la importancia del comportamiento alimentario en el estado de salud del individuo, ya que la actividad física por sí sola es insuficiente para mantener un peso corporal normal si el consumo de alimentos es mayor al gasto de calorías en la rutina diaria o de ejercicio.

Por otra parte, un porcentaje importante de hombres reportó problemas de sobrepeso y obesidad durante alguna etapa de la vida y entre sus familiares, dato que destaca el rol de la familia, ya que esta estaría siendo parte de la problemática en el sentido en que los diferentes miembros que la conforman pueden propiciar un ambiente favorecedor de la sobrealimentación y la inactividad física (Guzmán et al., 2010).

Adicional a lo anterior, el grupo con obesidad reportó mayor insatisfacción corporal, hallazgo que coincide con estudios previos (Sarwer, Thompson \& Cash, 2005; Weinberger, Kersting, Riedel-Heller \& Luck-Sikorski, 2016; Zawawi, 2014); sin embargo, también se ha sugerido que no todas las personas con obesidad presentan insatisfacción corporal (Schwartz \& Brownell, 2004), por lo que en una línea futura de investigación se sugiere determinar qué contribuye para que algunas personas con esta condición de peso desarrollen insatisfacción corporal.

Asimismo, en personas con obesidad se observaron altas puntuaciones en afecto negativo, como lo son la tristeza, la culpa, el temor, entre otras (Goldschmidt et al., 2014; Pasco, Williams, Jacka, Brennan \& Berk, 2013). Por su parte, el grupo con normopeso se diferenció de los de sobrepeso y obesidad en el funcionamiento físico, el rol físico, el funcionamiento social y el rol emocional, lo cual indica que los participantes con normopeso son capaces de realizar cualquier tipo de actividad física o social sin que sus emociones o su apariencia física los obstaculice, y en consecuencia manifiestan mejor calidad de vida; hallazgo que concuerda con distintos estudios previos (Busutil et al., 2017; Oliva, 2009; Pimenta, Bertrand, Mograbi, Shinohara \& Landeira-Fernández, 2015). Estos resultados reflejan que las personas con sobrepeso u obesidad no solo presentan afecciones de orden biológico, ya que su ajuste psicológico y social también se encuentra afectado por su condición corporal.

Otro de los hallazgos de esta investigación es que más del $60 \%$ de los participantes reportó tener estudios a nivel superior, y que, de la misma manera, el $67.95 \%$ de la muestra presenta sobrepeso u obesidad. Estos datos coinciden con lo reportado en la ENSANUT Medio Camino (Shamah et al., 2016), ya que en este se señala que siete de cada diez adultos mexicanos presentan sobrepeso u obesidad, y porque la muestra seleccionada realiza trabajo de oficina, es decir, uno de los más sedentarios - nivel terciario de gasto de energía- y que se asocia a la presencia de obesidad y ENT (Ramírez \& Agredo, 2012; Wanjek, 2005). Sin embargo, se esperaría que a mayor nivel educativo se tuviera mayor conciencia del cuidado de la salud, pero esto no se ve reflejado en los resultados. Una posible explicación a este hallazgo es que no hay una relación causal entre el nivel educativo y el cuidado de la salud, ya que también intervienen otros factores, como por ejemplo el acceso a alimentos saludables, el uso de áreas recreativas que fomenten la actividad física, e, incluso, la calidad del medio ambiente — contaminación atmosférica-, entre otras (Villar, 2011).

Adicionalmente, una de las consecuencias de la obesidad y sobrepeso es la presencia de ENT, sin embargo, en el presente estudio solo un porcentaje bajo $(20 \%)$ de la muestra reportó padecer hipertensión o diabetes. Este hallazgo llama la atención, ya que casi $70 \%$ de los empleados administrativos del sector salud tiene exceso de peso, pero menos de la cuarta parte de esta presenta comorbilidad médica. Explicaciones a este hallazgo son que la obesidad no siempre se asume como una enfermedad, $\mathrm{y}$, en consecuencia, las personas no asisten al médico para 
una revisión periódica de su salud, sino hasta presentar síntomas de ENT, o bien deciden no acudir al médico a pesar de presentar malestar y prefieren automedicarse para eliminar el síntoma temporalmente, sin recibir un diagnóstico formal sobre su padecimiento. Junto con a la presencia de ENT, también se observó la presencia de malos hábitos alimenticios, consumo de alcohol, tabaco e inactividad física, hallazgos que concuerdan con estudios previos (Escrivao, Oliveira, Taddei \& López, 2000; Miguel \& Niño, 2009; Reséndiz et al., 2015).

En consecuencia con tales resultados, la presente investigación destaca la urgencia de que en México se diseñen y establezcan programas que promuevan una cultura del cuidado de la salud, en donde se fomente una alimentación saludable y se mejoren las condiciones laborales para que se contribuya en la mejora de la calidad de vida no solo del personal administrativo, sino de la población general. Esto se justifica, adicionalmente, porque se calcula que el gasto anual que México invierte en la atención médica de ENT derivadas de la obesidad es de entre 78 mil y $101 \mathrm{mil}$ millones de pesos (mexicanos) y un costo indirecto por pérdida de productividad de entre 73 mil y 101 mil millones (Gutierrez et al., 2012); lo que significa un elevado costo de mantenimiento para los departamentos de salud. Adicional a esto, se debe tomar en cuenta que entre las principales causas de mortalidad en México se encuentran las ENT derivadas de la obesidad (INEGI, 2017b).

Cabe mencionar que los cuestionarios de autorreporte pudieron limitar la precisión de los datos obtenidos, por lo cual se sugiere que se entreviste a los participantes para complementar los hallazgos de la investigación. Además, la escala ATOP no cuenta con propiedades psicométricas en población mexicana, y aunque la consistencia interna en esta muestra de estudio fue buena, se recomienda que futuras investigaciones evalúen sus propiedades para tener mayor precisión en la medida. Por otra parte, este estudio buscó reflejar algunas de las consecuencias de la obesidad en trabajadores del sector salud, sin embargo, es necesario extender la investigación a variables que no se abordaron, como por ejemplo la conducta de atracón, de la cual se tiene conocimiento de su vínculo con la presencia de la obesidad (Hudson, Hiripi, Pope \& Kessler, 2007).

Finalmente, con este estudio se concluye, por una parte, que la presencia de conductas alimentarias anómalas puede ser consecuencia de la insatisfacción con la imagen corporal y la percepción de un mal funcionamiento físico y falta de vitalidad — es decir, de una pobre calidad de vida - y, por otra, que no existen diferencias en el IMC entre sexos, pero un alto IMC sí repercute en la calidad de vida de las personas.
Teniendo esto en cuenta, el presente estudio hace un llamado a la Secretaría de Salud para que emprenda campañas de salud entre sus trabajadores no solo a nivel nutricional, sino también a nivel conductual o cognitivo, para así coadyuvar con la disminución del gasto económico que ocasiona el tratamiento de la obesidad y de las patologías relacionadas tanto para la persona que la padece como para la institución, el país y la sociedad. Si bien la Secretaría de Salud ha implementado programas como "Reto Hazte Fuerte", "Reto 10000 pasos" y la "1. y 2. ${ }^{a}$ Evaluación del Índice de Condición Física" —que coordinó la Organización Mexicanos Activos y la Dirección de Recursos Humanos de la Secretaría de Salud Federal-, es relevante destacar que se le ha dado una nula importancia a la dimensión psicológica, por lo que con la presente investigación se intenta mostrar que también hay variables psicológicas que contribuyen al desarrollo y mantenimiento del sobrepeso y la obesidad; de ahí la necesidad de su incorporación en el diseño y aplicación de programas preventivos.

\section{Referencias}

Allison, D. B., Basile, V. C., \& Yurker, H. E. (1991). The measurement of attitudes toward and beliefs about obese persons. International Journal of Eating Disorders, 10(5), 599-607. doi:10.1002/1098-108X(199109)10:5<599::AIDEAT2260100512>3.0.CO;2-\#

Bell, S. K., \& Morgan, S. B. (2000). Children's attitudes and behavioral intentions toward a peer presented as obese: does a medical explanation for the obesity make a difference? Journal of Pediatric Psychology, 25(3), 137-145. doi: 10.1093/jpepsy/25.3.137

Bollado, J. G. (2014). Mitos en educación física y deporte: ¿reto superado o anclados en el pasado? Ribalta. Quaderns d'aplicació didáctica i investigación, 21, 111122. Recuperado de https://dialnet.unirioja.es/servlet/ articulo? codigo $=4815910$

Brechan, I., \& Kvalem, I. L. (2015). Relationship between body dissatisfaction and disordered eating: Mediating role of self-esteem and depression. Eating Behaviors, 17, 49-58. doi: 10.1016/j.eatbeh.2014.12.008

Bully, P., \& Elosua, P. (2011). Changes in body dissatisfaction relative to gender and age: The modulating character of BMI. Spanish Journal of Psychology, 4(1), 313-322. doi: 10.5209/rev_SJOP.2011.v14.n1.28

Busutil, R., Espallardo, O., Torres, A., Martínez-Galdeano, L., Zozaya, N., \& Hidalgo-Vega, A. (2017). The impact of obesity on health-related quality of life in Spain. Health and Quality of Life Outcomes, 15(1), 1-11. doi: 10.1186/ s12955-017-0773-y 
Campos-Nonato, I., Barquera, S., \& Aguilar, C. A. (2012). Efecto de la obesidad sobre otras enfermedades crónicas y la mortalidad. En J. A. Rivera, M. Hernández, C. A. Aguilar, F. Vadillo F., \& C. Murayama (eds.), Obesidad en México: Recomendaciones para una política de Estado (pp. 259278). México: Universidad Nacional Autónoma de México.

Casillas-Estrella, M., Montaño-Castrejón, N., Reyes-Velázquez, V., Bacardi-Gascón, M., \& Jiménez-Cruz, A. (2006). A mayor IMC mayor grado de insatisfacción de la imagen corporal. Revista Biomédica, 17(4), 243-249. doi: 10.32776/ revbiomed.v17i4.463

Cooper,P.J., Taylor,M.J.,Cooper,Z.,\&Fairburn,C.G.(1987). The developmentand validation of theBody ShapeQuestionnaire. International Journal of Eating Disorders, 6(4), 485494. doi: 10.1002/1098-108X(198707)6:4<485::AIDEAT2260060405>3.0.CO;2-O

De Zwaan, M., Petersen, I., Kaerber, M., Burgmer, R., Nolting, B., Legenbauer, T., ... Herpertz, S. (2009). Obesity and quality of life: a controlled study of normal-weight and obese individuals. Psychosomatics, 50(5), 474-482. doi: 10.1176/ appi.psy.50.5.474

Escrivao, M. A., Oliveira, F. L., Taddei, J. A., \& Lopez, F. (2000). Obesidad exógena en la infancia y adolescencia. Jornal de Pediatria, 76(3), S305-S310. Recuperado de https://www.pediatriaintegral.es/publicacion-2015-07/ obesidad-en-la-infancia-y-adolescencia/

Franco, K., Solorzano, M., Díaz, F. J., \& Hidalgo-Rassmussen, C. (Octubre, 2016). Confiabilidad y estructura factorial del Test de Actitudes Alimentarias (EAT-26) en mujeres mexicanas. Manuscrito presentado en el XXIV Congreso Mexicano de Psicología, Ciudad de México.

García, P. (2012). La alimentación de los mexicanos: Cambios sociales y económicos, y su impacto en los hábitos alimenticios. México: Cámara Nacional de la Industria de la Transformación.

Garner, D. M., Olmsted, M. P., Bohr, Y., \& Garfinkel, P. E. (1982). The Eating Attitudes Test: psychometric features and clinical correlates. Psychological Medicine, 12(4), 871878. doi: $10.1017 / \mathrm{s} 0033291700049163$

Gilbert-Diamond, D., Baylin, A., Mora-Plazas, M., \& Villamor, E. (2009). Correlates of obesity and body image in Colombian women. Journal of Women's Health, 18(8), 1145-1151. doi: 10.1089/jwh.2008.1179

Goldschmidt, A. B., Crosby, R. D., Engel, S. G., Crow, S. J., Cao, L., Peterson, C. B., \& Durkin, N. (2014). Affect and eating behavior in obese adults with and without elevated depression symptoms. International Journal of Eating Disorders, 47(3), 281-286. doi: 10.1002/eat.22188

Gutiérrez, J. P., Rivera-Dommarco, J., Shamah-Levy, T., Villalpando-Hernández, S., Franco, A., ... Cuevas-Nasu, L. (2012). Encuesta Nacional de Salud y Nutrición 2012. Resultados Nacionales. Cuernavaca, México: Instituto Nacional de Salud Pública.
Guzmán, R. M. E., del Castillo, A., \& García, M. (2010). Factores psicosociales asociados al paciente con obesidad. En J. A. Morales (ed.), Obesidad. Un enfoque multidisciplinario (pp. 201-218). México: Universidad Autónoma del Estado de Hidalgo.

Hudson, J. I., Hiripi, E., Pope, H. G., \& Kessler, R. C. (2007). The prevalence and correlates of eating disorders in the National Comorbidity Survey replication. Biological Psychiatry, 61(3), 348-358. doi: 10.1016/j.biopsych.2006.03.040

Instituto Mexicano del Seguro Social (IMSS). (Octubre, 2017). Calcula tu IMC. Recuperado de http://www.imss.gob.mx/ salud-en-linea/calculaimc

Instituto Nacional de Estadística y Geografía (INEGI). (Diciembre, 2016). Módulo de práctica deportiva y ejercicio físico. Recuperado de http://www.beta.inegi.org.mx/ proyectos/enchogares/modulos/mopradef/

Instituto Nacional de Estadística y Geografía (INEGI). (Ocrubre, 2017a). Indicadores de ocupación y empleo al tercer trimestre de 2017. Recuperado de http://www3.inegi. org.mx/sistemas/temas/default.aspx?s=est\&c=25433\&t=1

Instituto Nacional de Estadística y Geografía (INEGI). (Octubre, 2017b). Principales causas de mortalidad por residencia habitual, grupos de edad y sexo del fallecido. Recuperado de http://www.inegi.org.mx/est/contenidos/proyectos/regis tros/vitales/mortalidad/tabulados/ConsultaMortalidad.asp

Mäkinen, M., Puukko-Viertomies, L., Lindberg, N., Siimes, M. A., \& Aalberg, V. (2012). Body dissatisfaction and body mass in girls and boys transitioning from early to mid-adolescence: additional role of self-esteem and eating habits. BMC Psychiatry, 12(35), 1-8. doi: 10.1186/1471-244X-12-35

McGuinness, S., \& Taylor, J. E. (2016). Understanding body image dissatisfaction and disordered eating in midlife adults. New Zealand Journal of Psychology, 45(1), 4-12. Recuperado de https://www.psychology.org.nz/wp-content/ uploads/Body-Image-.pdf

Mellor, D., Fuller-Tyszkiewicz, M., McCabe, M. P., \& Ricciardelli, L. A. (2010). Body image and self-esteem across age and gender: A short-term longitudinal study. Sex Roles, 63(1), 672-681. doi: 10.1007/s11199-010-9813-3

Miguel, P. E., \& Niño, A. (2009). Consecuencias de la obesidad. Acimed, 20(4), 84-92. Recuperado de http://scielo.sld.cu/scielo. php?pid=S1024-94352009001000006\&script=sci_abstract

Nanguce, M., Ceballo-León, P., \& Álvarez-Reyes, N. (2015). Prevalencia de sobrepeso y obesidad en el personal de salud de la clínica de medicina familiar casa blanca del ISSSTE. Horizonte Sanitario, 14(2), 71-74. doi: https://doi. org/10.19136/hs.a14n2.943

Navarro, C., Venegas, U., Navarro, J. C., Corbalá, J., Cárdenas, V. M., \& Campos, A. (2005). Sobrepeso y obesidad entre mujeres profesionales de la salud. Ginecología y Obstetricia de México, 73(8), 401-406. Recuperado de https://www. medigraphic.com/pdfs/ginobsmex/gom-2005/gom058b.pdf 
Nieves-Ruíz, E. R., Hernández-Durán, A. G., \& AguilarMartínez, N. C. (2011). Obesidad en personal de enfermería de una unidad de medicina familiar. Revista de Enfermería del Instituto Mexicano del Seguro Social, 19(2), 87-90. Recuperado de https://www.medigraphic.com/pdfs/enfermeriaimss/eim-2011/eim112e.pdf

Oliva, J. (2009). Obesidad y calidad de vida relacionada con la salud. España: Departamento de Análisis Económico y Finanzas de la UCLM.

Organización Mundial de la Salud. (2010). Recomendaciones mundiales sobre actividad fisica para la salud. Suiza: Organización Mundial de la Salud.

Organización Mundial de la Salud (2014). 10 facts on obesity. Recuperado de http://www.who.int/features/factfiles/ obesity/en

Organización Mundial de la Salud (2017). Las 10 principales causas de defunción. Recuperado de http://www.who.int/ mediacentre/factsheets/fs310/es/

Organización para la Cooperación y el Desarrollo Económico. (2018). Obesity Update. Recuperado de http://www.oecd. org/healyh/obesity-update.htm

Palacios-Rodríguez, R. G., Munguía-Miranda, C., \& ÁvilaLeyva, A. (2006). Sobrepeso y obesidad en personal de salud de una unidad de medicina familiar. Revista Médica del Instituto Mexicano del Seguro Social, 44(5), 449-453. Recuperado de https://www.redalyc.org/ pdf/4577/457745535009.pdf

Pasco, J. A., Williams, L. J., Jacka, F. N., Brennan, S. L., \& Berk, M. (2013). Obesity and the relationship with positive and negative affect. Australian and New Zealand Journal of Psychiatry, 47(5), 477-482. doi: $10.1177 / 0004867413483371$

Pimenta, F. B. C., Bertrand, E., Mograbi, D. C., Shinohara, H., \& Landeira-Fernández, J. (2015). The relationship between obesity and quality of life in Brazilian adults. Frontiers in Psychology, 6(1), 1-7. doi: 10.3389/fpsyg.2015.00966

Ramírez, R., \& Agredo, R. A. (2012). El sedentarismo es un factor predictor de hipertrigliceridemia, obesidad central y sobrepeso. Revista Colombiana de Cardiología, 19(2), 7579. doi: 10.1016/S0120-5633(12)70109-2

Reséndiz, A. M., Hernández, S. V., Sierra, M. A., \& Torres, M. (2015). Hábitos de alimentación de pacientes con obesidad severa. Nutrición Hospitalaria, 31(2), 672-681. doi: 10.3305/nh.2015.31.2.7692

Robles, R., \& Páez, F. (2003). Estudio sobre la traducción al español y las propiedades psicométricas de las escalas de afecto positivo y negativo (PANAS). Salud Mental, 26(1), 69-75. Recuperado de https://www.redalyc.org/ pdf/582/58212608.pdf

Sarwer, D. B., Lavery, M., \& Spitzer, J. C. (2012). A review of the relationships between extreme obesity, quality of life, and sexual function. Obesity Surgery, 22(4), 668-676. doi: 10.1007/s11695-012-0588-1
Sarwer, D. B., Thompson, J. K., \& Cash, T. F. (2005). Body image and obesity in adulthood. Psychiatric Clinics of North America, 28(1), 69-87. doi: 10.1016/j.psc.2004.09.002

Schwartz, M. B., \& Brownell, K. D. (2004). Obesity and Body Image. Body Image, 1(1), 43-56. doi: 10.1016/ S1740-1445(03)00007-X

Shaheen, A., Kumar, H., Dev, W., Parkash, O., \& Rai, K. (2016) Gender Difference Regarding Body Image: A Comparative Study. Advances in Obesity, Weight Management \& Control, 4(4), 76-79. doi: 10.15406/aowmc.2016.04.00092

Shamah, T., Cuevas, L., Rivera, J., \& Hernández, M. (coord.) (2016). Encuesta nacional de salud y nutrición de medio camino 2016. Informe final de resultados. México: Instituto Nacional de Salud Pública.

Stewart A. L., Hays, R. D., \& Ware, J. E. (1988). The MOS short-form general health survey. Reliability and validity in a patient population. Medical Care, 26(7), 724-735. doi: 10.1097/00005650-198807000-00007

Thompson, J. K., Heinberg, L. J., Altabe, M., \& TantleffDunn, S. (1999). Exacting beauty: Theory, assessment, and treatment of body image disturbance. Washington: American Psychological Association.

Thompson, J. K., \& Smolak, L. (2001). Body image, eating disorders, and obesity in youth. Assessment, prevention and treatment. Washington: American Psychological Association.

Vázquez, R., Galán, J., López, X., Alvarez, G. L., Mancilla, J. M., Caballero, A., \& Unikel, C. (2011). Validez del Body Shape Questionnaire (BSQ) en mujeres mexicanas. Revista Mexicana de Trastornos Alimentarios, 2(1), 42-52. Recuperado de http://www.scielo.org.mx/scielo. php?script=sci_arttext\&pid=S2007-15232011000100005

Vázquez, R., Medina, K., López, X., López, C., Ortega, M., ... Rodríguez, R. (2017, octubre). Validación en mujeres mexicanas del Eating Attitudes Test (EAT-26): Análisis confirmatorio. Manuscrito presentado en el XXV Congreso Mexicano de Psicología, Puerto Vallarta, Jalisco.

Villar, M. (2011). Factores determinantes de la salud: Importancia de la prevención. Acta Médica Peruana, 28(4), 237-241. Recuperado de http://www.scielo.org.pe/scielo. php?script=sci_arttext\&pid=S1728-59172011000400011

Wanjek, C. (2005). Food at work. Workplace solutions for malnutrition, obesity and chronic diseases. Geneva: International Labour Office.

Watson, D., Clark, L. A., \& Tellegen, A. (1988). Development and validation of brief measures of positive and negativeaffect: the PANAS scales. Journal of Personality and Social Psychology, 54(6), 1063-1070. doi: 10.1037//0022-3514.54.6.1063

Weinberger, N., Kersting, A., Riedel-Heller, S. G., \& LuckSikorski, C. (2016). Body dissatisfaction in individuals with obesity compared to normal-weight individuals: A systematic review and meta-analysis. Obesity Facts, 9(6), 424-441. doi: 10.1159/000454837 
Zawawi, J. A. (2014). Relationships between body mass index, body image dissatisfaction and psychological distress among fitness center female users in Zarqa-Jordan. International Journal of Humanities and Social Science, 4(11), 109-130. Recuperado de http://www.ijhssnet.com/ journals/Vol_4_No_11_1_September_2014/12.pdf
Zúniga, M. A., Carrillo-Jiménez, G. T., Fos, P. J., Gandek, B., \& Medina-Moreno, M. R. (1999). Evaluation of health status using Survey SF-36: Preliminary results in Mexico. Salud Pública de México, 41(2), 110-118. Recuperado de https:// www.ncbi.nlm.nih.gov/pubmed/10343514 